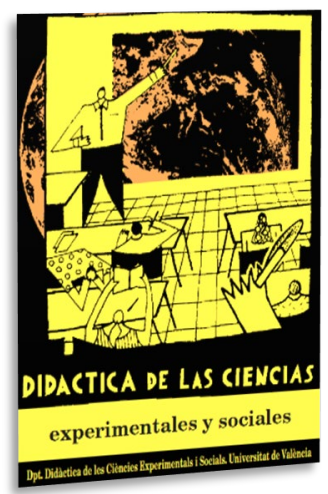

\title{
Reversión de los estereotipos de género en torno a la ciencia. Innovación en la enseñanza frente a los estereotipos de género sobre la ciencia
}

\author{
Reversal of gender stereotypes around science. \\ Innovation in teaching against gender \\ stereotypes about science
}

DOI: $10.7203 / D C E S .38 .15000$

\author{
María Luisa Mulero Martí \\ Universitat de València, maluimu@alumni.uv.es \\ Ángel San Martín Alonso \\ Universitat de València, asanmart@uv.es \\ ORCID iD: https://orcid.org/0000-0003-3565-4250
}

\begin{abstract}
RESUMEN: En este artículo se muestra el trabajo realizado en un centro escolar de Educación Primaria en la ciudad de Valencia cuyo objetivo de investigación es comprobar cómo, mediante una propuesta didáctica centrada en el alumnado de esta etapa educativa, se pueden cambiar los estereotipos de género que desde la sociedad se les transmiten, fenómeno que está cobrando relevancia en distintos ámbitos sociales y educativos, activando numerosos proyectos con finalidades semejantes a este. Así pues, los resultados más destacables se refieren a los aspectos organizativos del centro educativo y a los cambios impulsados por las actividades realizadas con motivo de El día Internacional de la mujer y la niña en la Ciencia.
\end{abstract}

Palabras Clave: mujeres, ciencia, igualdad, ámbito científico, Educación Primaria.

ABSTRACT: This article shows the work carried out at a Primary School in the city of Valencia to verify that gender stereotypes transmitted to Primary Education students by our society can be reversed through teaching proposals. This type of phenomena is gaining relevance in different social and educational fields and diverse projects are being carried out with the same aim. Thus, the most outstanding results refer to the School's organizational aspects and the changes promoted by the activities carried out during the International Day of Women and Girls in Science.

KEYWORDS: women, science, equality, scientific field, Primary Education.

Fecha de recepción: mayo de 2019

Fecha de aceptación: septiembre de 2019

Proyecto de innovación e investigación educativa «La ciència per a tots i totes» (código: 18AQ65IN002). Actividad convocada por la RESOLUCIÓN de 30 de mayo de 2017, del director general de Política Educativa de la Conselleria d'Educació, Investigació, Cultura i Esport. 


\section{INTRODUCCIÓN}

Hay un principio bueno que creó el orden, la luz y el hombre, y un principio malo que creó el caos, la oscuridad y la mujer.

(Pitágoras, 582- 507 a.c.)

Un primer punto de partida nos lo brinda la cita precedente, de parecido tenor a la que nos ofrece Charles Darwin, conocido por sus aportaciones sobre la evolución de las especies, pero no tanto por sus reflexiones en torno a la naturaleza de la mujer en su obra The Descent of Man and Selection in Relation to Sex (1987). A partir de este contexto cultural hacemos una primera aproximación a la situación que las mujeres han tenido respecto a los hombres a lo largo de los siglos. En el presente trabajo nos centramos en los estereotipos que vienen dificultando el que las mujeres accedan a la educación y a responsabilidades plenas en el ámbito científico.

Son muchas las referencias que durante estos últimos años se están realizando tanto en trabajos de divulgación, congresos, mesas redondas e incluso en los medios de comunicación, acerca de las desigualdades que tiene y han tenido las mujeres en el acceso y divulgación de la ciencia. Reflexiones al respecto se dan en una reciente publicación (García y Pérez, 2017), en la cual se analizan los obstáculos que han tenido que superar las mujeres científicas, detallando numerosas ocasiones en las que las mujeres han quedado a la sombra de hermanos, maridos, maestros, compañeros de investigación... otorgándoles sus aportaciones a la ciencia ya que investigaban en equipo y el trabajo de ellas permanecía oculto.

"En definitiva, la pregunta que nos queda tras este viaje es si nos encontramos ante ejemplos de mala ciencia o de ciencia al uso. Si mejorar la ciencia consistirá en eliminar los sesgos de género, si eso es posible, o si nos tendremos que replantear otras formas de hacer ciencia" (García y Pérez 2017: 241), reflexiones finales que se plantean las autoras al cuestionarse la objetividad en el contexto productor de la ciencia, siendo complejo evitar cualquier tipo de sesgos, ya se de género, raza o ideología.

Durante mucho tiempo la ciencia se ha considerado un pasatiempo para aficionados y las mujeres podía acceder a ella a través de colaboraciones con sus maridos o hermanos. Sin embargo, en el S.XIX comenzó a institucionalizarse, y las mujeres se vieron relegadas a un segundo plano, encontrando grandes dificultades para acceder al terreno científico (Saini, 2018). El primer acceso de las mujeres a universidades españolas se dio en 1868, viéndose restringido de nuevo su libre acceso en 1882 por medio de una Real Orden. Surgieron iniciativas educativas progresistas e innovadoras, fueron las realizadas por Rosa Sensat o María Baldo, ambas preocupadas por conseguir una educación integral de las niñas, que les proporcionara independencia económica. Voces a favor de reformar la situación legal de las mujeres, pero no fue hasta el S.XX cuando las reivindicaciones tomaron más fuerza gracias al apoyo de abogadas como Clara Campoamor o Matilde Huici.

Las mujeres tuvieron que esperar hasta el año 1910, para consolidar el libre acceso a las universidades y ver reforzados sus derechos con la creación de nuevas instituciones pedagógicas y nuevos métodos de enseñanza. Se fundó la Residencia de Señoritas con la finalidad de fomentar un trato natural y culturalmente equitativo entre hombres y mujeres, estimulando a las estudiantes a continuar sus estudios y prepararse para ingresar en las Facultades universitarias, consiguiendo de ese modo una paulatina incorporación de las mujeres en estudios universitarios.

Incluso contando con la actividad determinante para la consolidación de una élite intelectual moderna, como la Junta para Ampliación de Estudios e Investigaciones Científicas (JAE) creada en 1907, las mujeres encontraron durante este siglo dificultades para adentrarse en el terreno de las ciencias experimentales. A tal retroceso contribuyó de forma decisiva la Guerra Civil y en otras muchas ocasiones el matrimonio.

No obstante, desde finales del siglo pasado se incrementó el interés por reescribir la historia de la ciencia, favoreciendo la inclusión de mujeres a través de movimientos feministas cuyo objetivo era 
entrelazar feminismo e historiografía. Pues "no se trata solo de acumular nombres de mujeres sino de analizar cómo sus historias de vida configuran en cada momento histórico las prácticas científicas y con ellas la ciencia misma" (Santesmases, Cabré i Pairet, Ortiz, 2017).

Por ello, actualmente existen proyectos como Inspiringirls o el de Hypatia, que promueven la incorporación de las niñas y las mujeres al mundo de la ciencia y la tecnología a través de proyectos pedagógicos y culturales, para aumentar la autoestima y las vocaciones científicas en edad escolar.

Este es el punto de partida del presente trabajo de investigación, en el que se trata de proponer otro modo de hacer ciencia en la Educación Primaria mediante una propuesta didáctica de investigación-innovación. En la propuesta implementamos una serie de actividades didácticas planificadas en un proyecto de innovación cooperativo para todos los cursos de la educación primaria, y posteriormente se han analizado los resultados obtenidos. Desde la perspectiva adoptada, se analizan los sesgos de género que puedan darse en el tratamiento de la ciencia en el contexto escolar, con tal de eliminar esas desigualdades, tan difíciles de modificar ya que no disponemos actualmente de un conocimiento científico que incluya la experiencia y el saber de las mujeres (Marbà y Solsona, 2012).

En definitiva, se trata de ofrecer una visión diferente desde el currículum, incluidos los libros de texto, en el que faltan referentes femeninos, para trazar una manera distinta de transmitir la historia de la ciencia al alumnado en el aula de Educación Primaria. Nuestro objetivo pues, es construir un saber diferente del que ha sido tradicional en los libros de texto y en el desarrollo del currículo, para comprobar si es posible contribuir a modificar los estereotipos que se mencionan en los estudios recientes sobre el tema, así como en el proceso de indagación e investigación que se ha seguido en la siguiente propuesta.

\section{MARCO TEÓRICO}

Los estereotipos (García y Pérez, 2017), son creencias sobre un grupo concreto, ampliamente compartidas por la sociedad, que pueden ser positivos, neutros o negativos, suponiendo en este último caso discriminaciones susceptibles de ser directas o indirectas. Los estereotipos de género, reflejan ideas normativas de lo que es considerado la feminidad o la masculinidad, creencias esencialistas que crean dualismos $\mathrm{u}$ opuestos binarios. Estos estereotipos a veces se basan en realidades o características que en algún tiempo han tenido diversos grupos, pero tienden a perpetuarse incluso cuando esa realidad demográfica ha cambiado. En concreto, se está investigando cómo los sesgos de género o los estereotipos conllevan una falta de vocaciones científicas en las adolescentes, así como una falta de alfabetización científica en la ciudadanía o una definición de metas y expectativas diferentes para ambos sexos.

Desde los primeros pensadores como Aristóteles, Platón, Descartes o el ya mencionado Darwin, se han hecho referencias o se ha descrito al hombre y a la mujer con diferencias sustanciales, infravalorando a las mujeres y posicionándola un escalón por debajo del hombre, tanto física como intelectualmente atribuyendo estas diferencias como biológicas. Al género masculino se le atribuyen características tales como la estabilidad emocional, objetividad y racionalidad, carácter dominante, aptitudes intelectuales, eficacia... todas ellas susceptibles de ser aplicables también a la ciencia, mientras que a las mujeres se les atribuyen cualidades como intuición, dependencia, inestabilidad emocional, irracionalidad...

Históricamente se ha hablado también de esta inferioridad de las mujeres especialmente en el periodo menstrual, por la debilidad o histeria que se genera debido al desajuste hormonal. Supuestos que Leta Hollingworth desmontó con su tesis doctoral en 1914 Functional Periodicity: An experimental study of the mental and motor habilities of women during the menstruation, afirmando que este periodo no podía suponer una discriminación para las mujeres en su acceso al mundo laboral ni, en este caso, al científico.

Al interpretar en multitud de ocasiones estas diferencias como biológicas e innatas, se naturalizan las desigualdades de género, las esferas separadas, convirtiéndolas en inamovibles y en 
un "debe ser" (Hare-Mustin y Marecek, 1994). A lo largo de la historia se ha infravalorado a las mujeres debido a diversas causas: biológicas, psicológicas, médicas, sociológicas... y se han ido transmitiendo estas creencias a través de la familia, los iguales, los medios de comunicación y las instituciones educativas, creando expectativas concretas y diferentes sobre el comportamiento que deben tener hombres y mujeres, niños y niñas.

Sin embargo, tanto investigadores como instituciones están haciendo un esfuerzo para conseguir mejorar el acceso y la visibilidad de las mujeres en la ciencia a través de algunos proyectos e investigaciones como los citados. En concreto, desde la Fundación Española para la Ciencia y la Tecnología (FECYT) se ha contribuido a analizar la presencia de las mujeres en el ámbito científico mediante estudios e investigaciones que ponían de manifiesto la multitud de aportaciones que éstas han hecho. Recientemente la Unidad de Mujeres y Ciencia (UMyC) junto con el Ministerio de Ciencia, Innovación y Universidades, han publicado el informe Cientificas en Cifras-2017, donde se ofrecen estadísticas e indicadores de desigualdad de género en la formación y profesión científica, mostrando la escasa presencia de mujeres que a día de hoy se sigue dando en los cargos de gobierno de las universidades y Órganos Públicos de Investigación, así como el "efecto tijera" y el techo de cristal que se produce en la carrera investigadora en la que únicamente hay un $21 \%$ de mujeres en las cátedras de universidad y un $25 \%$ entre el profesorado de investigación. Éstos son datos que hay que ayudar a cambiar comenzando desde la formación inicial en la Educación Primaria.

Así mismo, en otro estudio titulado La situación de las mujeres investigadoras en el sistema educativo español de ciencia y tecnología (FECYT, 2005:53), extraían conclusiones referentes a las microdesigualdades que se van generando a partir de "prácticas informales" que consiguen que "las mujeres disponen de menos recursos presupuestarios, les es más difícil obtener los servicios del personal de apoyo, se ubican en los despachos que están más lejos... y no disponen de un grupo de mentores equivalente al de sus colegas varones a quien pedir asesoramiento y apoyo".

A partir de éste y otros informes similares, consiguieron establecer legalmente algunas de las reivindicaciones en torno a los derechos para las mujeres y la igualdad de género, elaborando la ORDEN PRE/525/2005, de 7 de marzo, por la que se da publicidad al Acuerdo de Consejo de Ministros en el que se adoptan medidas para favorecer la igualdad entre mujeres y hombres. En concreto, en el punto cuarto sobre investigación "se acuerda crear una unidad específica de «Mujer y Ciencia» para abordar la situación de las mujeres en las instituciones investigadoras y mejorar su presencia en ellas".

Igualmente se creó un plan de igualdad de género en la carrera científica de la agencia estatal del CSIC (2007-2009) "para estimular, para avanzar, para caminar por la senda de quienes han apostado por la I+D como motor de progreso", estableciendo medidas para promover la igualdad entre hombres y mujeres, mostrándose como modelo de buenas prácticas para la comunidad científica. Prueba de ello es que en noviembre de 2017 se nombró a Rosa Menéndez presidenta del CSIC, siendo la primera mujer que ocupa este cargo desde que se fundó la agencia, tras haber ocupado varios puestos institucionales y de gestión a lo largo de su carrera.

No obstante, hoy se sigue indagando sobre estas diferencias entre hombres y mujeres en otros ámbitos. Un ejemplo son los informes PISA, en los que mediante las pruebas que se aplican al alumnado de secundaria de distintos países de la OCDE, se extraen "conclusiones muy relevantes" de las capacidades que tienen según el género en la competencia lectora, matemática y científica. Si bien es cierto que en la actualidad se ha observado un descenso en el interés de los adolescentes por las carreras del ámbito científico, puede deberse tal y como muestran algunos estudios, al bagaje cultural que arrastran cuando llegan a los quince/dieciséis años, pues predispone a los adolescentes ante las distintas materias que les ofrece la institución escolar (Crawford y Capps, 2016).

En el informe Rocard que la Comisión Europea encargó al antiguo primer ministro francés en 2009, se promueve una reorientación pedagógica de la enseñanza de las ciencias tanto en las escuelas de educación Primaria como de Secundaria, se introducen recomendaciones para impulsar las líneas de actuación que traten aspectos de género "incluida la presentación de modelos de roles para las 
niñas en forma de éxito de mujeres científicas, ingenieras y empresarias." Inciden en la importancia de incrementar la confianza y la participación de las niñas en las tareas escolares científicas en todas las etapas y niveles del sistema educativo. Además insisten en la importancia del papel del profesorado en esta renovación científica que debe darse desde las instituciones educativas, examinando proyectos como Pollen para la enseñanza primaria o Sinus-Transfer para la Secundaria, a través de los cuales aseguran que se ha producido una renovación en la práctica de la ciencia, basada más en la indagación.

Desde el Ministerio de Educación se intentó ayudar al profesorado en general a renovar sus prácticas educativas mediante algunas propuestas. Tales como analizar la situación concreta de la educación científica en España, difundiendo datos relevantes sobre la relación entre las mujeres y la ciencia, además de presentar actividades concretas aplicadas al currículo de Primaria. Sin olvidar tampoco la importancia de los materiales o recursos que se utilizan en el aula, cobrando un papel primordial los libros de texto de las editoriales.

Destacamos sobre el particular que se dedica aproximadamente un $75 \%$ del tiempo en el aula a la revisión de los libros de texto, así como el 90\% del tiempo a los deberes que se realizan en casa (Simón, 2000). Y la realidad es que estos libros de texto se presentan como un dispositivo globalizador y unificador de la materia escolar, pero no solo fomenta el dominio de ese saber en concreto, sino de todo un código de símbolos sociales (Subirats, 2006).

Así pues, resaltamos los datos extraídos del estudio comparado (Blanco, 2016: 14), en el que concluye que "los hombres gozan de una vida profesional variada, representada en figuras de cocineros, alcaldes, caballeros, médicos, profesores... siempre con responsabilidad y, en la mayoría de los casos como jefes o, en su defecto, con cierto poder en las acciones que realizan." Observa además que "en ninguna ocasión aparecen trabajando en el hogar. Por su parte, las mujeres son dependientas, secretarias, profesoras (bajo los tradicionales estereotipos)...; y aparecen en muchos casos trabajando en el hogar...".

En este sentido, cabe destacar que tampoco los libros de texto juegan un papel a favor de las mujeres (López-Navajas, 2014), pues de varias investigaciones podemos extraer datos que confirman la baja presencia de mujeres en todas las asignaturas del currículo de la Educación Secundaria, llegando a ser "su presencia anecdótica y su ausencia, sistemática" ya que no están incluidas en el cuerpo del texto que conforman las páginas del libro de texto, ni son protagonistas de la información que se transmiten, contando con porcentajes globales de aparición únicamente del 12,8\%. Estos datos conllevan a extraer conclusiones sobre la escasa presencia de las mujeres en los libros de texto, y afirmaciones que evidencian " la ocultación de las mujeres en el relato de la historia, su ausencia de la tradición cultural y la prueba de la falta de autoridad social que se les concede, que busca y consigue que ignoremos la genealogía del conocimiento femenino" (López-Navajas, 2014: 20).

De aquí la necesidad de seguir analizando esta situación y de repensar los contenidos académicos, tanto en los libros de texto como en cualquier modalidad de consumo cultural que esté al alancance del alumnado, tanto dentro como fuera del aula. A pesar de haber disminuido las representaciones sexistas en los estudios recientes, sigue subestimándose la contribución de las mujeres a la cultura y la sociedad (Blanco, 2004), al tiempo que se silencian sus necesidades y expectativas.

Lo cual invita a preguntarse: ¿qué criterios sigue el profesorado para seleccionar una u otra editorial? Y un poco más allá, ¿son válidos los criterios del profesorado a la hora de seleccionar los distintos recursos? En esta línea se podrían proponer otras opciones, por ejemplo, la creación de sellos de calidad para otorgar a los libros coeducativos y que orienten al profesorado a la hora de decantarse por unos materiales u otros (Vaíllo, 2017).

Es por ello que desde la Educación Primaria, tanto en el currículum oficial como en las prácticas de aula, se deben realizar pequeñas acciones que fomenten la igualdad tanto en expectativas de la capacidad que tienen en el ámbito científico, como en el acceso real de ambos géneros. Diversos autores (Raviolo, Ramírez y López, 2010) proponen realizar en la formación inicial del profesorado 
actividades como: 1) analizar distintos ejemplos de modelos para identificar las características que tienen en común; 2) construir y reformular modelos, reflexionando sobre sus propósitos; o 3) explicar y predecir fenómenos concretos con modelos construidos por los alumnos, considerando las limitaciones que presentan.

En una dirección semejante presentamos en el siguiente epígrafe las propuestas didácticas realizadas en un centro público de la ciudad de Valencia, encaminadas a visibilizar la presencia de las mujeres en la ciencia mediante actividades ajenas a los libros de texto. Pretendemos analizar cómo se pueden cambiar los estereotipos de género que le llega al alumnado de educación Primaria y que condicionan en la adolescencia la elección de una determinada carrera universitaria. Cuestión sobre la que, hasta el momento, no se han realizado demasiados estudios orientados a evaluar los resultados de la intervención con tales propósitos en la citada etapa educativa.

\section{Planteamiento del estudio}

\subsection{Diseño metodológico}

El estudio se centra en unas propuestas didácticas enmarcadas dentro de la jornada El Día Internacional de la Mujer y la Niña en la Ciencia, en un colegio público de Educación Infantil y Primaria de la ciudad de Valencia. El objetivo principal es analizar cómo los estereotipos en torno a la ciencia que el alumnado hace suyos a través del consumo cultural fuera y dentro del centro escolar, se pueden modificar mediante la aplicación de unas propuestas didácticas concretas encaminadas a visibilizar la importancia del papel de las mujeres en el ámbito científico. Por tanto, el objeto de estudio consistirá en analizar las producciones que el alumnado realiza antes y después de las actividades organizadas con mujeres científicas, así como detallar las manifestaciones verbales y no verbales que expresa el alumnado en torno a su interés por la ciencia.

En la propuesta didáctica han participado los cuatrocientos sesenta y cinco discentes del centro, a través de las distintas actividades que se han organizado para cada uno de los cursos de la Educación Primaria. Las actividades se han diseñado tomando como referente el área de Ciencias Naturales, al mismo tiempo que se les ha intentado dar un planteamiento transversal, vinculándolas con otras áreas del currículum.

El centro participaba desde el curso anterior en el desarrollo y evaluación de un proyecto de innovación educativa La ciencia per a tots $i$ totes, subvencionado por la Conselleria de Educación. Lo promueven dos maestras de primaria del centro escolar que imparten las áreas de ciencias y comprometidas con la renovación del laboratorio escolar y de las prácticas científicas que se realizaban en el aula. Tanto en los objetivos generales del proyecto como en los objetivos específicos, se incluye la necesidad de destacar el papel de las mujeres en el desarrollo de la ciencia, para contribuir a la igualdad en todos los ámbitos.

Con esta finalidad, se plantearon las jornadas en las que se propusieron varios talleres (Tabla 1) en las que participaron tres científicas en activo y de distintas especialidades, siendo acompañadas durante las actividades realizadas por los correspondientes docentes e investigadores. La propuesta se desarrolló en el mes de febrero de 2018. Previamente a la puesta en práctica, durante el mes de enero de 2018, docentes y científicas se reunieron para pensar en la temática que se iba a desarrollar, centrando siempre el foco en intentar hacer visible el papel de las mujeres en la ciencia; en el interés pedagógico de los distintos talleres planteados, así como en la necesidad de reflexionar sobre el desarrollo de los mismos en los meses posteriores. De modo que se dedicaron varias sesiones a debatir en torno a cómo percibe el alumnado de Educación Primaria el trabajo desempeñado por las mujeres en la ciencia, cuáles son sus creencias y los estereotipos de género. Sesiones que fueron observadas y registradas, como indicaremos más adelante.

Para analizar el material recogido adoptamos un enfoque de estudio de casos múltiples (Stake, 2005), porque de esta manera podíamos desentrañar mejor los testimonios de las diferentes sesiones 
y todo ello en el contexto del centro escolar que implementa el proyecto. De acuerdo con planteamientos adecuados para este tipo de estudios (Strauss y Corbin, 1990), se diseñó el repertorio de técnicas e instrumentos de análisis de documentos y testimonios en las siguientes fases: en un primer momento la observación, en segundo lugar entrevistas no estructuradas y por último cuestionarios de valoración sobre las experiencias realizadas. Además, también se han extraído datos de los ejercicios de expresión escrita que el alumnado realizó posteriormente, así como de las composiciones gráficas generadas a raíz de las actividades propuestas con el fin de compararlas con las expresiones plásticas que habían compuesto antes de iniciar la aplicación del proyecto de innovación mencionado.

Los datos extraídos en las observaciones se registraron mediante unas rejillas de evaluación previamente validadas con las científicas que iban a participar en la iniciativa así como con el profesorado del centro educativo; estando estas rejillas relacionadas con las categorías iniciales susceptibles de estudio, así como fotografías realizadas con el móvil y grabación mediante cámara de vídeo de las actividades propuestas. Los cuestionarios iniciales planteados al alumnado se realizaron mediante el programa informático QuestionPro, y las entrevistas no estructuradas se grabaron a través de la app Notas de Voz. Además, la síntesis de toda la información obtenida se realizó mediante el software para análisis de datos cualitativos Atlas.ti 8, realizando una triangulación de los registros obtenidos, así como con las rejillas de evaluación incluidas en el proyecto de innovación La ciencia per a tots $i$ totes.

Mediante las rejillas que se elaboraron a partir de las categorías establecidas para analizar los dibujos que el alumnado realizó previamente con la finalidad de observar la idea que tienen en torno a la ciencia, y poder extraer de ahí los estereotipos de género que se han generado a través del consumo cultural, se analizaron también los dibujos que se les solicitó elaboraran una vez realizadas todas las actividades didácticas, para poder compararlos y establecer conclusiones sobre el éxito de las tareas propuestas y la consecución del objetivo principal del proyecto.

TABLA 1. Experiencias planificadas durante las jornadas

\begin{tabular}{|c|c|c|}
\hline Acción & Ámbito & Alumnado \\
\hline Ambientación de aulas & Centro & 465 \\
\hline Renovación bibliográfica & Centro & 465 \\
\hline Murales biográficos & Aula/Centro & 40 \\
\hline La vida de Marie Curie & Aula & 43 \\
\hline Charla con una astrónoma & Aula & 120 \\
\hline Actividad científica 1 & Aula & 120 \\
\hline Actividad científica 2 & Aula & 80 \\
\hline Actividad científica 3 & Aula & 120 \\
\hline$\overline{\text { Total }}$ & & $\begin{array}{l}\text { Ha participado todo el } \\
\text { alumnado del centro } \\
\text { escolar. }\end{array}$ \\
\hline
\end{tabular}

Fuente: Elaboración propia

\subsection{Descripción de las jornadas}

En este punto se describen las acciones realizadas en el centro educativo, cuyos objetivos eran: a) identificar los conocimientos que el alumnado tiene acerca del papel de las mujeres en el ámbito científico y cómo repercuten en el desarrollo de sus representaciones en torno a la ciencia en función de sus intervenciones en el centro escolar; b) reconocer la importancia y visibilidad que las mujeres deberían tener en el desarrollo del conocimiento científico; c) analizar la repercusión que pueda tener 
el proceso didáctico y pedagógico planteado a través de las propuestas organizativas realizadas en el centro, así como las propuestas didácticas en las aulas de los distintos niveles.

En cuanto a la organización del centro escolar, se tuvieron en cuenta aspectos muy diversos, entre otros la ambientación de las distintas estancias de centro, especialmente el laboratorio. Se estamparon citas de científicas tomadas de Jane Goodall y un árbol en el que se van introduciendo distintas científicas tanto de la antigüedad como actuales, según se van presentando y conociendo en las actividades de aula.

También a la hora de mostrarles recursos bibliográficos, tanto en el laboratorio como en la biblioteca escolar, se utilizaron técnicas e instrumentos de datos, como los ya mencionados cuestionarios compuestos de preguntas abiertas con la finalidad de conocer sus intereses y motivaciones, poniendo a su alcance estos manuales así como especialmente libros en los que se hace hincapié en visibilizar las aportaciones de las mujeres a la ciencia. Así pues, se pusieron al alcance del alumnado manuales como: Ada magnifica cientifica, Supermujeres Superinventoras, Me llamo... Marie Curie, Cuentos de buenas noches para niñas rebeldes, Yo Jane, Mujeres de ciencia, Las chicas son de ciencia...

Por otro lado, se propusieron actividades específicas para desarrollar en las clases de los distintos niveles. Por ejemplo el alumnado de sexto de Educación Primaria realizó murales que se colocaron en los pasillos del centro escolar, sobre la vida y trabajo de distintas mujeres científicas (Imagen 1).

IMAGEN 1: Murales con las mujeres científicas

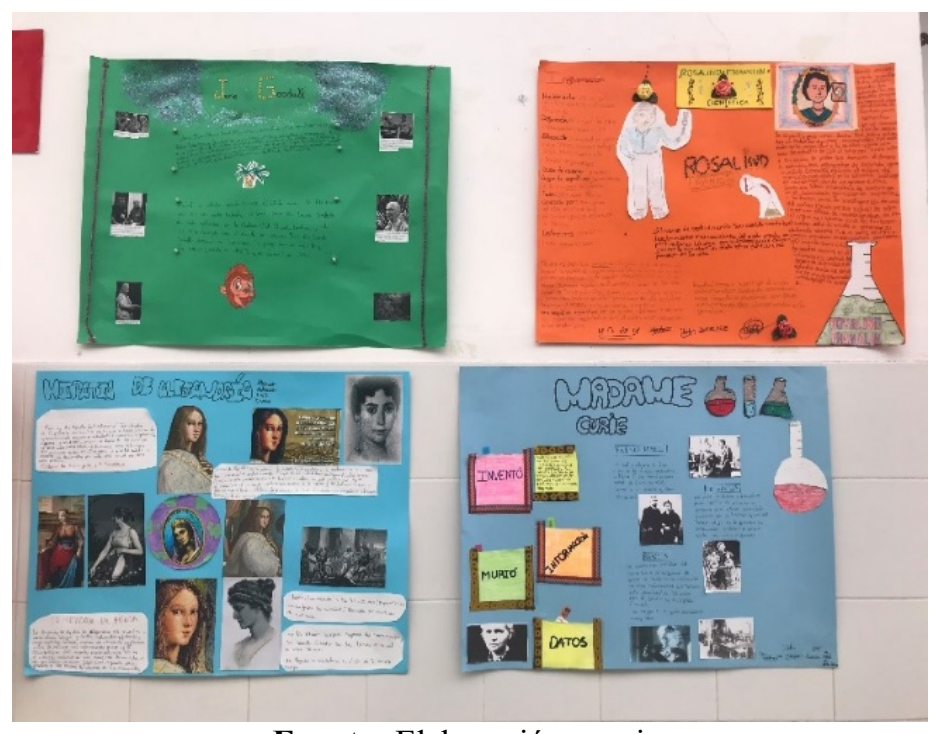

Fuente: Elaboración propia.

En quinto de Educación Primaria leyeron la biografía de Marie Curie (ya que se habían comprado diversos ejemplares para la biblioteca escolar), y realizaron varias actividades a su alrededor, como crear la línea del tiempo con los datos más destacados de su vida o reescribir su biografía. Con el apoyo de las TIC crearon también una presentación de Powtoon con los aspectos más significativos de su vida, con la finalidad de mostrarlo al alumnado de cursos inferiores y contribuir así a su difusión.

Con motivo de las celebraciones del 11F: Día Internacional de la Mujer y la Niña en Ciencia, cabe resaltar el conjunto de actividades realizadas. El día 7, organizado por la Comisión Mujer y Astronomía de la Sociedad Española de Astronomía, se realizó una Charla con una Astrónoma, además se habilitó un chat entre las aulas del colegio para que alumnas y alumnos intercambiaran dudas y opiniones. Así pudieron conversar con Amelia Ortiz, que trabaja en el Observatorio de 
Valencia y se dedica a divulgar material escolar científico, más concretamente en adaptarlo a alumnado que pueda tener algún tipo de discapacidad para favorecer su acceso al conocimiento científico. Les resultó muy interesante ya que pudieron realizar muchas preguntas y observar trabajos que Amelia había realizado a través de los enlaces que nos iba proporcionando, además de invitarles a visitar su lugar de trabajo.

Los días 8 y 9 visitaron el centro tres científicas, una de ellas antigua alumna de la escuela, aspecto que incrementó su interés al tratarse de un referente cercano con el que poder identificarse. Para despertar su interés, previamente se expuso en el centro información sobre su trabajo así como algunas fotografías que ellas mismas habían remitido (Imagen 2). También en las distintas aulas de las clases y en la puerta del laboratorio, se expuso un horario con las actividades que los distintos cursos iban a realizar durante esas jornadas.

IMAGEN 2. Científicas que participaron en las jornadas escolares sobre ciencia.

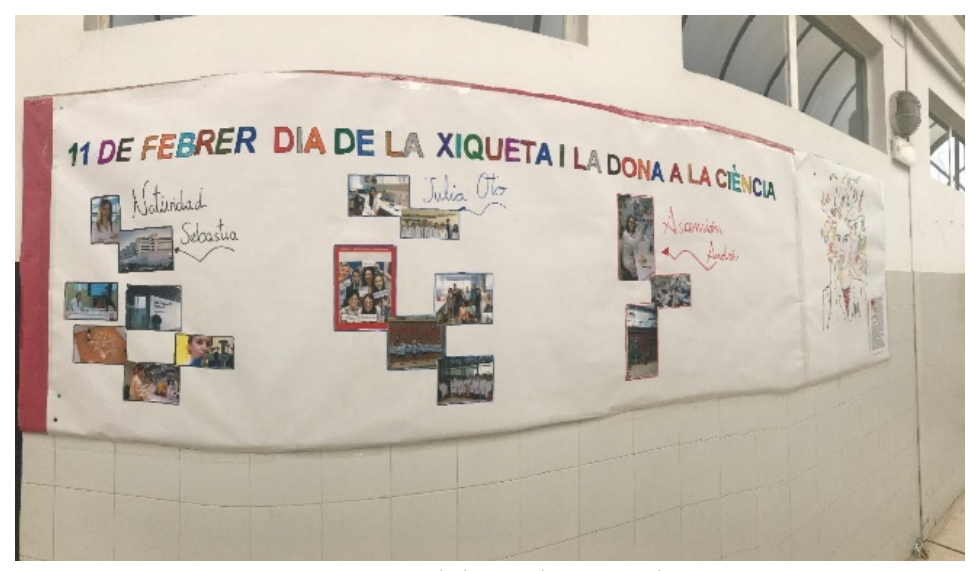

Fuente: Elaboración propia.

Por otra parte, el día 8 se realizaron actividades en distintas aulas de forma simultánea. Natividad Sebastià Fabregat (Imagen 3), científica del Instituto de Investigación Sanitaria de La FE. Le contó al alumnado de cuarto y quinto la investigación que llevaba a cabo para descubrir nuevas técnicas no invasivas con la que descubrir enfermedades, contando para ello con la ayuda de la perra Yuca. Al alumnado de primero y segundo, les estuvo explicando otra investigación que lleva a cabo relacionada con las radiaciones: cómo les podían afectar las radiaciones del sol, cómo se utilizaban las radiaciones en el día a día en el hospital y cómo las utilizaba ella a través de muestras de sangre en el microscopio.

IMAGEN 3. Natividad Sebastià

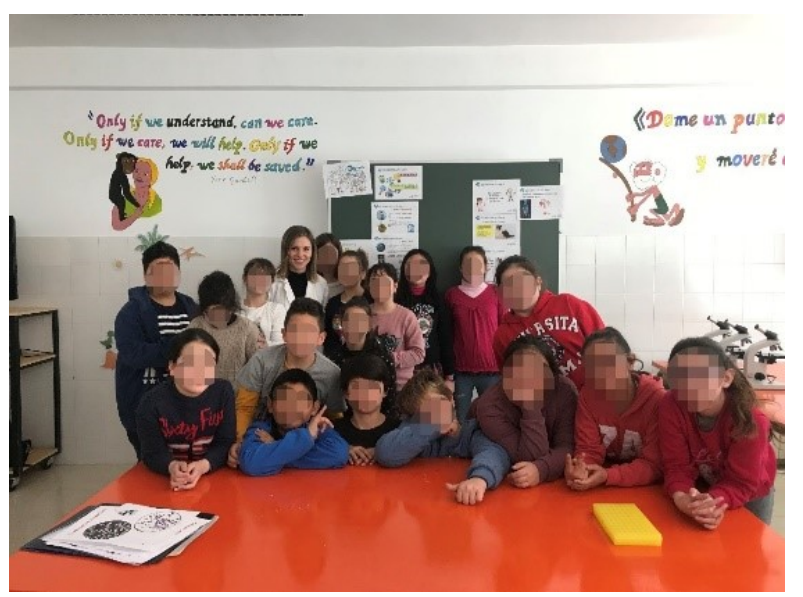

Fuente: Elaboración propia 
Mientras que Julia Oto (Imagen 4), antigua alumna de la escuela e investigadora también en el Instituto de Investigación Sanitaria de La Fe, les habló de sus estudios sobre diferentes enfermedades y les ayudó a extraer su ADN al alumnado de quinto y sexto. Con ellos tratan de entender cómo funcionan y así encontrar curas, vacunas, diagnósticos...

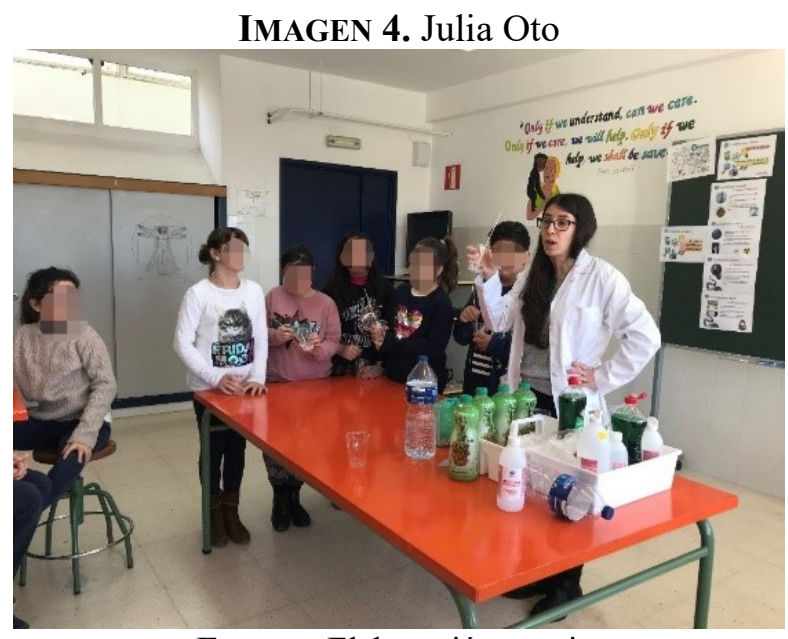

Fuente: Elaboración propia.

Por otro lado, el día 9 de febrero, Ascensión Andrés (Imagen 5), biotecnóloga en la Universidad de Valencia, pronunció una charla al alumnado de tercero, cuarto, quinto y sexto de primaria explicándoles dónde trabajaba, qué investiga su equipo de trabajo... Complementó su exposición con distintas experiencias, en las que podían extraer los pigmentos de las plantas, comprobar las bacterias que hay en las manos, observar la evolución de distintos insectos...

IMAGEN 5. Ascensión Andrés

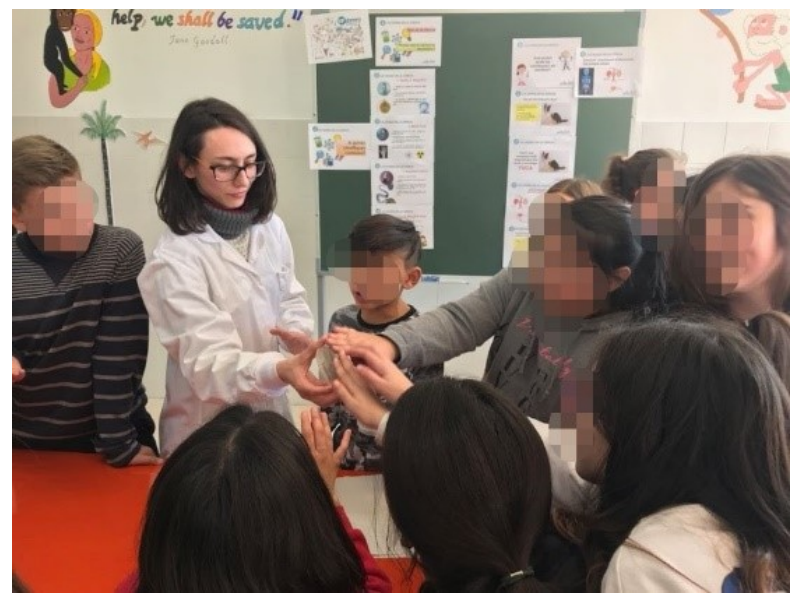

Fuente: Elaboración propia.

\section{RESULTADOS}

La implementación del proyecto generó impactos muy distintos entre el alumnado del centro y también en la ambientación y organización del colegio. No obstante, en este apartado nos ocuparemos principalmente de los resultados más relevantes obtenidos en el proceso de aplicación del mismo, resultados organizados a partir de las diferentes estrategias diseñadas en las primeras fases del proceso (Tabla 2). 


\subsection{Propuestas organizativas de centro}

Una primera observación a destacar es el incremento de la asistencia del profesorado a las sesiones de coordinación del proyecto. La distribución de carteles y murales por el centro, en segundo lugar, fue muy bien valorada por los estudiantes, quienes mostraron a través de las entrevistas semiestructuradas gran interés en conocer un poco más sobre la historia de las mujeres que veían en murales y pancartas. Especial interés manifestaron por el mural que anunciaba la visita de tres científicas a la escuela (Imagen 2), deteniéndose la mayoría del alumnado a ver sus nombres, profesión y preguntando al profesorado sobre su visita (ver observación 1 en Tabla 2).

En cuanto a la renovación bibliográfica, la bibliotecaria del centro constató un aumento en el préstamo de libros relacionados con la ciencia, y en concreto con los nuevos ejemplares que se habían colocado en la sección de novedades y que tenían temáticas relacionadas con mujeres científicas; así como en los manuales colocados en el laboratorio escolar (ver registro 1 en Tabla 2).

Respecto a los murales biográficos y lecturas de científicas como Marie Curie, el alumnado mostró entusiasmo en las respuestas a las preguntas planteadas en la entrevista no estructurada, además de observarse por parte de los docentes y los investigadores una mejor predisposición a la hora de realizar las tareas, haciendo un trabajo de indagación individual en primer lugar, posteriormente de puesta en común en grupo y finalmente elaborando el producto final, que podía ser consultado tanto por sus compañeros de aula como por el resto de alumnado del centro (ver cuestionario 1 en Tabla 2).

TABLA 2. Resultados obtenidos en la investigación.

\section{Técnica de recogida de datos Extracto de evidencias}

Observación 1

Registro 1
En general el alumnado se detenía a consultar el mural, incluso en las salidas a casa en las que se suelen marchar con rapidez y hablando entre ellos.

El préstamo de manuales relacionados con el ámbito científico se vio incrementado en un $30 \%$ respecto a las anteriores semanas o meses.

Fuente: Elaboración propia.

\subsection{Propuestas didácticas en las aulas}

En cuanto a las actividades realizadas en las distintas aulas, sería destacable la gran acogida por parte de todo el alumnado independientemente de la edad. Tras hacer un análisis, se concluyó que las actividades propuestas estaban adaptadas a la edad e intereses del alumnado, por lo que fue fácil atraer su atención y conseguir un nivel muy alto de motivación (ver registro 2 en Tabla 3). Así lo reflejaba el alumnado, tanto en su expresión verbal como no verbal (ver observación 2 y entrevista 1 en Tabla 3). 
Con carácter diferencial cabe resaltar el entusiasmo puesto por el alumnado, especialmente entre las niñas, que al ver testimonios de mujeres científicas, que incluso habían estudiado en el mismo centro educativo en el que estaban ellas, se planteaban preguntas a ellas mismas y al profesorado sobre su posible futuro encaminado al ámbito científico. También, destacable que por su propia iniciativa, los más pequeños realizaron dibujos en los que les daban las gracias y les mostraban a las distintas científicas, cuánto les había gustado las actividades realizadas. Incluso un grupo de quinto de primaria, decidió enviar una carta a una de ellas, en agradecimiento a todo lo que les había mostrado.

TABLA 3. Extracto evidencias tras las jornadas realizadas a nivel de aula.

\begin{tabular}{|c|c|}
\hline Técnica de recogida de datos & Extracto de evidencias \\
\hline Registro 2 & $\begin{array}{l}\text { La valoración de las actividades por parte del } \\
\text { equipo docente fue muy positiva, proponiendo su } \\
\text { repetición el próximo curso escolar. } \\
\text { Las actividades les han aportado otro punto de vista } \\
\text { distinto al habitual, en otro espacio, situaciones } \\
\text { diferentes, tiempo para practicar, vincular los } \\
\text { conocimientos adquiridos con la vida real de forma } \\
\text { muy positiva. Se ha conseguido además incorporar } \\
\text { en estas jornadas, los diferentes contenidos } \\
\text { estudiados en las áreas de ciencias, con la práctica } \\
\text { en el laboratorio. }\end{array}$ \\
\hline Observación 2 & $\begin{array}{l}\text { El rostro del alumnado reflejaba ilusión y ganas de } \\
\text { aprender cuando miraban a las científicas. Se podía } \\
\text { ver tanto durante la realización de las actividades } \\
\text { como en las fotografías tomadas. }\end{array}$ \\
\hline Entrevista 1 & $\begin{array}{l}\text { Lo que ocurre es que cuando estudiamos ciencias } \\
\text { en clase solo lo hacemos con el libro, es aburrido y } \\
\text { no aprendemos tanto. No nos dejan utilizar } \\
\text { materiales. A mí me han gustado mucho las } \\
\text { actividades de las científicas y menos la escucha de } \\
\text { un profesor/a hablando durante horas. }\end{array}$ \\
\hline Entrevista 2 & $\begin{array}{l}\text { ¿Yo podría ser una científica como ella? ¿Qué } \\
\text { tendría que estudiar? }\end{array}$ \\
\hline
\end{tabular}

Fuente: elaboración propia.

En relación con el proceso didáctico y pedagógico de los talleres, tanto docentes como estudiantes y científicas, valoraron satisfactoriamente la realización de todas las sesiones, acercando la realidad científica y el trabajo desempeñado por cada una de ellas, más allá de la mera información que pueden extraer de los libros de texto. Los docentes subrayaron que estas actividades científicas enmarcadas dentro de las jornadas, rompieron con el estereotipo de género en el que suele vincularse al hombre con todo aquello relacionado con la ciencia, aportando una visión más amplia y apoyando así a la consecución de los objetivos didácticos establecidos en el currículum de toda etapa la educación primaria, como por ejemplo: "valorar y respetar la diferencia de sexos y la igualdad de derechos y oportunidades entre ellos" o "fomentar la igualdad efectiva de derechos y oportunidades entre hombres y mujeres, analizar y valorar críticamente las desigualdades existentes e impulsar la igualdad real y la no discriminación de las personas con discapacidad". Rechazar los estereotipos que 
supongan discriminación entre hombres y mujeres. Además, vieron enriquecedora esta formación para el propio profesorado ya que podrán aplicar estas dinámicas en el aula de ciencias y potenciar el método científico.

El alumnado consideró muy positivamente los cuestionarios finales para que valoraran la realización de estas propuestas metodológicas, otorgándoles en un $94,8 \%$ de los casos la máxima puntuación puesto que tal y como anotaron en las observaciones, consideraron que les beneficia porque permanecen más atentos, concentrados y activos. Además, despierta su interés por las disciplinas científicas al tener referentes cercanos con los que poder identificarse, tomándolos como ejemplo de lo que podrían llegar a hacer o conseguir en un futuro. Por otra parte, el 97\% del alumnado valoró con la máxima puntuación la información aportada por las científicas en las charlas, ya que comprobaron que investigaban sobre temas de interés para la sociedad en general y para ellos en particular, pudiendo realizar prácticas concretas en las que observar su propio ADN o comprobar que los perros podían ayudar en las investigaciones científicas, aspecto que motivó al alumnado y sobre el que realizaron muchas preguntas a la científica.

\section{CONSIDERACIONES FINALES}

En virtud de los objetivos formulados más arriba y que han orientado el estudio del caso de implementación de un proyecto para remover los estereotipos de género en la ciencia, es posible avanzar una primera conclusión en el sentido que desde bien pequeños el alumnado participa de ellos. Pero no es menos cierto, que cuando se trabaja en las aulas con proyectos que capten su atención es posible identificar y actuar antes tales estereotipos. Nuestro estudio proporciona evidencias sobre la toma de conciencia del alumnado a propósito del papel tan importante pero invisible que han tenido las mujeres en el avance científico. Hemos comprobado que el proyecto de actividades experimentado contribuyó a mitigar los estereotipos de género que tiene el alumnado de Educación Primaria en torno a la ciencia, así como la capacidad de despertar vocaciones científicas en las niñas mediante la muestra de modelos cercanos a ellas, presentándoselos como ejemplos tangibles y próximos a su realidad. No obstante, para conseguir una verdadera desactivación, debe abordarse de forma transversal desde todas las áreas curriculares y niveles docentes. Es necesario rehacer el sistema de valores y actitudes que se transmiten, es decir, repensar los contenidos educativos para eliminar cualquier tipo de connotación sexista y conseguir que el currículum prescrito refleje las aportaciones de las mujeres a la sociedad y a la cultura. No únicamente a través de efemérides o actuaciones aisladas, sino normalizando estos contenidos a través de la práctica diaria.

El análisis de las producciones artísticas del alumnado posteriores a la realización de las jornadas, mediante las rejillas orientadas a identificar las ideas previas del alumnado en torno a la presencia de las mujeres en la ciencia, ratificaron los datos obtenidos mediante la observación, las entrevistas y los cuestionarios. Inicialmente el 51\% del alumnado representó a hombres en actividades científicas y tan solo el $15 \%$ a mujeres; mientras que en el análisis posterior aumentó significativamente el número de niñas que representaban a mujeres científicas, alcanzando una representación del $46 \%$ del total del alumnado.

Los resultados de este estudio avalan la necesidad de diseñar e implementar actividades de formación para Primaria que se ajusten a la necesidad de hacer visible el papel de las mujeres en la ciencia. Se pretende así favorecer que el alumnado pueda tener un conocimiento más global y acceder al ámbito científico en igualdad de condiciones, contribuyendo de esta manera a aminorar la brecha de género en el ámbito científico. Tal y como plantea (Raviolo et al., 2010), el profesorado incluyó en las propuestas de mejora tanto del centro, como del proyecto de innovación mencionado anteriormente, la necesidad de reformular los modelos que se venían implementando en el centro escolar en torno al estudio de la ciencia, así como la revisión de la concreción curricular tanto en las áreas científicas como en el resto de las áreas del currículo. 
Los resultados arrojados tras la implementación de nuestro proyecto, son muy positivos, pero sin duda todavía muy limitados. Hemos descubierto qué actividades le resulta de interés al alumnado, cómo estimular su sentido crítico a través de la cartelería en las paredes del centro, pero es preciso seguir avanzando con nuevos estudios que además amplíen la muestra de centros. Es también importante conseguir el apoyo institucional, tanto a los centros educativos dotándoles de recursos para poder llevar a cabo proyectos de mejora y de innovación, como a los docentes en activo que quiere implementar nuevas estrategias metodológicas. Apoyar el desarrollo de estudios sobre proyectos de innovación como el planteado aquí, diseñando instrumentos de recogida de información más sutiles que los empleados en este estudio, lo que sin duda requiere la asignación de recursos para avanzar en la igualdad de oportunidades también en el ámbito científico.

\section{Referencias}

Alcalá Cortijo, P., Corrales Rodrigáñez, C. y López Giráldez, J. (2009). Ni tontas ni locas: las intelectuales en el Madrid del primer tercio del siglo XX. Madrid: FECYT.

Blanco García, N. (2004). El saber de las mujeres en la educación. XXI Revista de Educación, 6, 4353.

Blanco García, M. E. (2016). Representaciones y expresiones de género en los libros de texto de educación primaria. Estudio comparado de los cursos lectivos 1994/1995- 2014/2015. Recuperado de https://www.fes-sociologia.com/representaciones-y-expresiones-de-genero-enlos-libros-de-texto-de-ed/congress-papers/2319/ [10 de octubre de 2018].

Cervelló Collazos, J. (coord.) (2009). Educación científica "ahora": el informe Rocard. Madrid: Ministerio de Educación Cultura y Deporte.

Crawford, B. y Capps, D. (2016). What knowledge do teachers need for engaging children in science practices? En J. Dori, Z. Mevarech y D. Baker (eds.). Cognition, Metacognition and Culture in STEM Education. Nueva York: Springer.

Delord, G. y Porlán, R. (2018). Del discurso tradicional al modelo innovador en enseñanza de las Ciencias: obstáculos para el cambio. Didáctica de las ciencias experimentales y sociales, 35, 77-90. DOI: 10.7203/DCES.35.12193

Everet, S., Otto, C. y Luera, G. (2009). Preservice elementary teachers' growth in knowledge of models in a Science capstone course. International Journal of Science and Mathematics Education, 7, 1201-1225. DOI: 10.1007/s10763-009-9158-y

Fundación Española para la Ciencia y la Tecnología (2007). La situación de las mujeres investigadoras en el Sistema Español de Ciencia y Tecnología. Fundación Española para la Ciencia y la Tecnología. Madrid: FECYT. Recuperado de https://www.fecyt.es/es/publicacion/mujer-y-ciencia-la-situacion-de-las-mujeresinvestigadoras-en-el-sistema-espanol-de [14 de abril de 2018].

García Dauder S. y Pérez Sedeño E. (2017). Las 'mentiras' científicas sobre las mujeres. Madrid: Catarata.

Hare-Mustin R. y Marecek J. (1994). Marcar la diferencia: psicología y construcción de los sexos. Barcelona: Herder.

Leta Stetter Hollingworth (1914). Functional Periodicity: An Experimental Study of the Mental and Motor Abilities of Women During Menstruation. Teachers College, Columbia University Contributions to Education.

López Navajas, A. (2014). Análisis de la ausencia de las mujeres en los manuales de la ESO: una genealogía de conocimiento ocultada. Revista de Educación, 363, 282-308. DOI: 10.4438/1988-592X-RE-2012-363-188

Marbà-Tallada, A. y Solsona-Pairó, N. (2012). Identificación e interpretación de las posibles desigualdades formativas en ciencias de chicos y chicas en la educación obligatoria y el 
bachillerato. Revista Cultura y Educación, 24(3), 289-303. DOI: $10.1174 / 113564012802845659$

Olivos, T. (2011). La cultura de la evaluación y la mejora de la escuela. Perfiles Educativos, 33(131), 116-13. Recuperado de http://www.scielo.org.mx/scielo.php?script=sci_arttext\&pid=S0185-26982011000100008 $[10$ de enero de 2018].

ORDEN PRE/525/2005, de 7 de marzo, por la que se da publicidad al Acuerdo de Consejo de Ministros por el que se adoptan medidas para favorecer la igualdad entre mujeres y hombres.

Raviolo, A., Ramírez, P. y López, E. (2010). Enseñanza y aprendizaje del modelo científico a través de analogías. Revista Eureka de Enseñanza y Divulgación de las Ciencias, 7(3), 581- 612. DOI: 10.25267/Rev_Eureka_ensen_divulg_cienc.2010.v7.i3.01

Saini. A. (2018). Inferior. Madrid: Círculo de Tiza.

Sancho-Gil, J. M., y Correa-Gorospe, J. M. (2015). Aprender a enseñar: la constitución de la identidad del profesor en la Educación Infantil y Primaria. Movimento, 22(2), 471-484. DOI: $10.22456 / 1982-8918.58298$

Santesmases, M., Cabré i Pairet, M. y Ortiz, T. (2017). Feminismos biográficos: aportaciones desde la historia de la ciencia. Revista Arenal, 24(2), 379-40.

Simón, Ma E. (2000). Tiempos y espacios para la coeducación en el harén pedagógico. Barcelona: Graó.

Stake, R. (2005). Multiple Case Study Analysis. New York: The Guilford Press.

Strauss, A. y Corbin, J. (1990). Basics of Qualitative Research. California: Sage

Subirats Martori, M. (2006). La educación de las mujeres: de la marginalidad a la coeducación. Propuestas para una metodología de cambio educativo. En Género y Currículo. Aportaciones del género al estudio y práctica del currículo (pp. 229-255). Madrid: Akal.

Torres, J. y Vasconcelos, C. (2017). Desarrollo y validación de un instrumento para analizar las visiones de los profesores sobre modelos. Revista Eureka sobre Enseñanza y Divulgación de las Ciencias, 14(1), 181-198. Recuperado de: http://hdl.handle.net/10498/18855

Vaíllo, M. (2016). La investigación sobre libros de texto desde la perspectiva de género: ¿hacia la renovación de los materiales didácticos? Tendencias pedagógicas, 27, 97-124. DOI: $10.15366 / \mathrm{tp} 2016.27 .003$

\section{CÓMO CITAR ESTE ARTÍCULO}

Mulero, M. L. y San Martín, A. (2020). Reversión de los estereotipos de género en torno a la ciencia. Innovación en la enseñanza frente a los estereotipos de género sobre la ciencia. Didáctica de las ciencias experimentales y sociales, 38, 81-96. DOI: 10.7203/DCES.38.15000. 
